\title{
Introduction to the OQE special issue on Numerical Simulation of Optoelectronic Devices (NUSOD'11)
}

\author{
Wei Lu • Aldo Di Carlo • Lukas Chrostowski • \\ Mauro Pereira • Slawek Sujecki
}

Published online: 13 May 2012

C) Springer Science+Business Media, LLC. 2012

This is the special issue of 11th International Conference on Numerical Simulation of Optoelectronic Devices (NUSOD'11) in Optical and Quantum Electronics. The following papers have been presented on the NUSOD'11, peer-reviewed, and finally accepted by Optical and Quantum Electronics. The chairs of NUSOD'11 are Aldo Di Carlo and Matthias auf der Maur from University of Rome, and Joachim Piprek form NUSOD Institute. The NUSOD'11 was hold in Rome, Italy, 5-8 September, 2011. The published papers in this issue report on recent progresses in numerical simulations of the semiconductor lasers, light emitting diodes, photodetectors, photonic crystals, novel optical materials and devices, integrated systems, and so on.

W. Lu (凶)

Shanghai Institute of Technical Physics, Chinese Academy of Sciences, Shanghai, China e-mail: luwei@mail.sitp.ac.cn

\author{
A. D. Carlo \\ University of Rome "Tor Vergata", Rome, Italy \\ L. Chrostowski \\ University of British Columbia, Vancouver, BC, Canada
}

M. Pereira

Sheffield Hallam University, Sheffield, UK

S. Sujecki

University of Nottingham, Nottingham, UK 